\title{
Leucemia-linfoma de células T do adulto no Brasil: epidemiologia, tratamento e aspectos controversos
}

\author{
Adult T cell leukemia-lymphoma in Brasil: epidemiology, treatment and \\ controverstial aspects
}

Fernanda Azevedo Silva, ${ }^{1}$ Ernesto Meiss, ${ }^{2}$ ane de Almeida Dobbin ${ }^{2}$ e

Maria do Socorro Pombo de Oliveira ${ }^{3}$

\section{Resumo}

A leucemia-linfoma de células T do adulto (LLCTA) é uma neoplasia de linfócitos T maduros, associada à infecção pelo vírus linfotrópico de células T humanas do tipo I (H TLV-I). LLCTA ocorre mais freqüentemente em regiões onde a infecção pelo H TLV-I é endêmica, como no Japão e ilhas caribenhas, porém casos esporádicos já foram descritos em regiões não-endêmicas para infecção por HTLV-I. N o Brasil, as formas clínicas mais reconhecidas são as formas agudas e linfomatosas. Um fato interessante da coorte brasileira é a ocorrência desta doença em crianças e jovens (2-21 anos). Rio de Janeiro e Salvador são as cidades com maior ocorrência de casos diagnosticados, seguidos de Recife e São Paulo. LLCTA nas formas mais agressivas não responde aos tratamentos quimioterápicos convencionais com insucesso em termos de sobrevida $>5$ anos. No entanto, nos casos que receberam a consolidação do tratamento com a-interferon (alFN) associado a zidovudine ( $A Z T$ ) como esquema de manutenção de remissão, demonstraram melhor sobrevida. Recentemente o tratamento com anti-CD 25, apesar de estar em fase experimental, vem apresentado resultados promissores. Com o crescente reconhecimento da LLCTA dentre as doenças linfoproliferativas no Brasil, tornam-se necessários estudos terapêuticos diferenciados desta doença. Procuramos neste artigo descrever as características clínico-epidemiológicas dos casos brasileiros, e com a revisão da literatura sobre os resultados terapêuticos no tratamento do LLCTA, pretendemos propor um estudo com fins terapêuticos utilizando drogas moderadoras biológicas e anti-retrovirais.

Palavras-chave: leucemia-linfoma aguda de células T associada a H TLV-I; quimioterapia; epidemiologia; HTLV-I; Brasil.

${ }^{1}$ M édica. M estranda em H ematologia, U niversidade Federal do Rio de Janeiro, Laboratório de M arcadores Celulares Serviço de $\mathrm{H}$ ematologia.

${ }^{2}$ Serviço de $\mathrm{H}$ ematologia, H ospital do Câncer I, Instituto N acional de Câncer.

${ }^{3}$ Laboratório de $\mathrm{M}$ arcadores $\mathrm{C}$ elulares, Serviço de $\mathrm{H}$ ematologia, $\mathrm{H}$ ospital do $\mathrm{C}$ âncer I,Instituto $\mathrm{N}$ acional de $\mathrm{C}$ âncer. Enviar correspondência para M S.P.O. H C I, Praça Cruz Vermelha 23, 70 andar; 20230-130 Rio de Janeiro, RJ - Brasil. E-mail: mpombo@inca.gov.br

Recebido em setembro de 2002. 


\section{Abstract}

Adult T-cell leukemia/lymphoma (ATLL) is a malignant disease of T-cell mature lymphocyte associated to human T-cell lymphotropic virus type I (HTLV-I). Although ATLL frequently occurs in regions where HTLV-I infection are endemic, such as Japan and Caribbean islands, however sporadic cases have been described in HTLV-I non endemic areas. In Brazil, the most recognized clinical form of AT LL are acute and lymphoma subtypes The occurrence of AT LL in children and young patients was an interesting and distinct demographic features observed in the Brazilian cohort. Rio de Janeiro and Salvador cities where locations with the majority of cases registered, followed by Recife and São Paulo cities. The most aggressive clinical forms of AT LL are unresponsive to conventional chemotherapy protocols with poor rate of 5 years survival. $\mathrm{H}$ owever in cases who interferon-a and ziovudine were used as consolidation treatment the overall results demonstrated a better survival rate. Recently the alternative treatment using anti-CD 25 showed promising results, even though in experimental protocols. With the increasing recognition of ATLL in Brazil, it has been necessary to apply different therapeutic protocols for this disease. In this manuscript we described the clinical and epidemiological characteristics of the Brazilian cases and a review of literature regarding therapeutic approaches of ATLL seeking new insights and manage ment of biological modifiers and retroviral drugs.

Key words H TLV-I associated acuteT-cell leukemia-Iymphoma; chemotherapy; epi demiology; H T LVl; Brazil.

\section{INTRO DUÇÃO}

\section{Características clínicas e laboratoriais}

A leucemia-linfoma de células $T$ do adulto ( LLCTA) é uma neoplasia de linfócitos T maduros, relacionada à infecção pelo vírus linfotrópico de células $T$ humanas do tipo I (HTLV-I). Além do LLCTA, outras doenças como paraparesia espástica tropical e mielopatia associada a H TLV-I (TSP/H AM ), uveíte e dermatiteinfecciosa na infância fazem parte do espectro clínico da infecção por H TLV-I. O correm mais freqüentemente no Japão, onde LLCTA foi descrita pela primeira vez em 1977.1,2 N o Brasil a infecção pelo H TLV-I é considerada en dêmica, porém com baixo índice de prevalência $(0,4 \%-1,8 \%)$ na população geral, quando comparado com os índices do Japão (3\% - 16\%).3.,4 As regiões com maior número de casos diagnosticados são Rio de Janeiro, Salvador, Recife e São Paulo, dados demonstrados após análise de uma série de 238 casos coletados ao longo de oito anos do registro de casos de LLCTA no Brasil. $^{5}$

$$
\text { As formas clínicas do LLcTA }
$$

caracterizam-se em quatro subtipos: leucêmico agudo, linfoma, crônico e soldeing (forma oligo-sintomática). Além destes subtipos bem definidos por critérios clínicolaboratoriais, existe um estado limítrofe entre indivíduos assintomáticos e LLCTA, que é denominado como fase pré- LLCTA. N esta fase assintomática existe a presença de linfócitos atípicos circulantes que podem desaparecer espontaneamente e a doença é definida pela monoclonalidade da inserção proviral do HTLV-I no linfócito T CD 4+. ${ }^{6,7}$

$\mathrm{N}$ as formas clínicas mais agressivas do LLCTA (agudo e linfoma), o paciente apresenta-se com performance status (PS) comprometido, apresenta a síndrome tumoral caracterizada por linfoadenomegalias, lesões viscerais múltiplas (hepatoesplenomegalia e infiltração pulmonar), lesões de pele e lesões ósseas. H ipercalcemia causada pelo aumento da reabsorção óssea pelos osteoclastos pode ocorrer em $50 \%$ dos casos. 0 s pacientes com LLcTA são geralmente imunodeficientes, têm predisposição constante às infecções oportunísticas, sejam elas bacterianas, fúngicas, parasitárias ou virais. ${ }^{2,8,9} \mathrm{~A}$ sobrevida 
na grande maioria dos casos não ultrapassa 12 meses mesmo quando submetido a tratamento quimioterápico agressivo. A forma linfomatosa é a mais difícil de se caracterizar como LLCTA, devido à semelhança com os linfomas de um modo geral e principalmente pela ausência de um padrão histológico arquitetural patognomônico. Só quando o paciente cursa com lesões extranodais ou evolui com hipercalcemia é que a forma linfomatosa é diagnosticada [após sorologia reativa para $H T L V-I]$. Q uando o paciente cursa com formas smoldering ou crônica (não existe massa tumoral), os sintomas são mais inconsistentes com o tipo da doença maligna. $\mathrm{N}$ a grande maioria dos casos o diagnóstico de LLCTA só é feito durante a agudização do quadro.

$\mathrm{N}$ a série de casos brasileiros, $36 \%$ dos pacientes apresentaram alterações clínicas decorrentes de hipercal cemia como primeiros sinais de LLCTA, ou seja, taquicardia ou arritmia cardíaca, sonolência com confusão mental, letargia, diminuição do fluxo urinário e insuficiência renal. Por outro lado, após a confirmação diagnóstica do LLCTA, foi comum encontrar a referência de lesões de pele persistentes, de longa duração e quase sempre refratárias aos tratamentos dermatológicos. $\mathrm{N}$ a maioria destes casos 0 envolvimento cutâneo predominante caracterizava-se por lesões eritrodérmicas, pápulas, macroplacas, ou leões tumorais cutâneas de longa evolução.

0 exame morfológico das células linfóides muitas vezes é o primeiro sinal a despertar para o diagnóstico do LLCTA. Os linfócitos são caracterizados por um acentuado pleomorfismo celular, irregularidades nucleares e condensação de cromatina nuclear variável (Figura 1). As células mais típicas da LLCTA são linfócitos de médio tamanho com núcleos polilobulados (flower cell). 0 citoplasma é freqüentemente escasso e o núcleo irregular pode apresentar esboços de nucléolos. As células grandes são semelhantes a imunoblasto com citoplasma basofílico e cromatina nuclear delicada, com nucléolos proeminentes e aberrantes. É importante ressaltar que na forma smoldering a proporção de células anormais é inferior a 3\% e, nestes casos, as irregularidades nucleares são muitas vezes semelhantes às células cerebriformes hipercromáticas encontradas na micose fungóide. Por ser uma doença de origem periférica, os aspirados de medula óssea na sua grande maioria apresentam pequena infiltração de linfócitos convolutos anormais. As dosagens de cál cio sérico e de-hidrogenase lática (D H L) são indispensáveis na avaliação dos pacientes, pois hipercalcemia pode ser uma complicação fatal nestes pacientes com LLcTA aguda. D H L apresenta-se alterada em cerca de $80 \%$ dos casos na apresentação da doença sendo importante na caracterização entre os subtipos crônicos e smoldering conforme Tabela 1. Apesar de ainda ser utilizado para fins de pesquisa clínica, a dosagem sérica da interleucina IL-2 vem demonstrando ser um excelente indicador do status clínico e apresenta-se elevada nos pacientes com LLCTA nas formas aguda e linfomatosa. ${ }^{10}$

Figura 1. Aspectos morfológicos dos linfócitos naLLCTA, individualizando as diversas formas dos núcleos celulares que caracterizam a LLCTA. Letra A e C figuras á esquerda representam as formas típicas de flower cell.

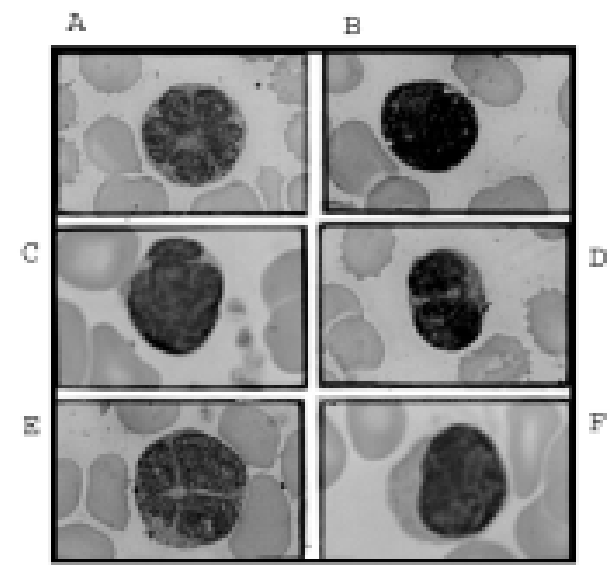

Em relação ao perfil imunofenotípico, as células da LLCTA expressam marcadores de células T como CD 2, CD 7, mCD 3, CD 5, CD 4+/CD 8- além de antígenos de ativação 
celular CD 38, CD 30+/-, H LAD r e CD 25+. Existe uma correlação importante entre os altos níveis de células em atividade proliferativa medidas pela quantidade de Ki-67 intracitoplasmática, com a rápida progressão da doença nas fases crônicas para fase aguda. ${ }^{11,12}$

Tabela 1. Características clínicas e laboratoriais que definem os critérios para classificação dos subtipos clínicos de LIcTA.

\begin{tabular}{|c|c|c|c|c|}
\hline & Smoldering & Crônico & Linfoma & Agudo \\
\hline Sorologia HTLV-I+ & + & + & + & + \\
\hline Leucócitos $\times 10^{9} / \mathrm{I}$ & $<4$ & $=4^{(1)}$ & $<4$ & $*$ \\
\hline Linfócitos anormais & $<5 \%$ & $+($ (II) & $=1 \%$ & $+($ (II) \\
\hline Flower cells & Ocasional & $\sim 10 \%$ & Não & + \\
\hline $\mathrm{DHL}$ & $=1.5 \mathrm{~N}$ & $=2.0 \mathrm{~N}$ & * & $*$ \\
\hline Cálcio sérico & $<10.7$ & $<10.7$ & * & $*$ \\
\hline Histologia linfoma $\mathrm{T}$ & Não & $*$ & + & $*$ \\
\hline Pele & $* *$ & $*$ & $*$ & * \\
\hline Pulmão & *** & * & * & * \\
\hline Linfoadenopatia & Não & $*$ & Sim & $*$ \\
\hline Fígado & Não & $*$ & * & $*$ \\
\hline Baço & Não & $*$ & * & $*$ \\
\hline Sistema nervoso central & Não & Não & * & * \\
\hline Osso & Não & Não & * & $*$ \\
\hline Ascite & Não & Não & $*$ & $*$ \\
\hline Efusão pleural & Não & Não & * & * \\
\hline
\end{tabular}

Abreviações:*não é essencial; **não é essencial se outros itens forem preenchidos, mas a prova histológica de lesão maligna é requerida; (I) acompanhada por linfocitose $\mathrm{T}(3.5 \times 109 / /$ ou mais $)$.
(PCRI). I sto porque o padrão de monoclonalidade é estabelecido através da digestão do D N A humano com endonucleases específicas e pela visualização de bandas que representam os fragmentos do H TLV-I inserido no genoma humano. ${ }^{14-16} \mathrm{~A}$ endonuclease ECO-RI divide o padrão de integração do HTLV-I em 3 tipos: completo, múltiplo e defectivo. Estes padrões são utilizados como indicadores de resposta terapêutica e como fatores prognósticos. ${ }^{15,16}$

\section{LEUCEMIA-LIN FO MA DE CÉLULAS T NO BRASIL}

A partir de um projeto sobre incidência e patogênese de LLCTA no Brasil, foi criado um registro de casos de LLCTA com base nãopopulacional, no Laboratório de $\mathrm{M}$ arcadores Celulares - Instituto $\mathrm{N}$ acional de $\mathrm{C}$ âncer (IN CA), Rio de Janeiro (1992-1999). Este estudo teve como objetivo identificar as possíveis diferenças regionais de LLCTA no Brasil, bem como comparar o perfil celular e molecular dos casos incidentes com aqueles descritos no Japão. O s casos avaliados durante a elaboração deste estudo foram resultantes da notificação e colaboração de vários pesquisadores. O s pacientes foram identificados em diversos Centros M édicos e registrados de acordo com os dados clínicos enquanto amostras de sangue eram enviadas para confirmação diagnóstica no IN CA. $N$ este estudo, 110 pacientes foram diagnosticados no Rio de Janeiro, dos quais 80 foram registrados de forma consecutiva, e 30 casos retrospectivos foram resgatados, após sorologia para H TLV-I; 49 pacientes foram diagnosticados no $D$ epartamento de Patologia e M edicina Interna da Universidade Federal da Bahia em Salvador; 30 pacientes foram consecutivamente diagnosticados e tratados no Departamento de $\mathrm{H}$ ematologia do HEM O PE, Recife-Pernambuco; 18 pacientes foram consecutivamente diagnosticados e tratados no Departamento de $\mathrm{H}$ ematologia da Escola Paulista de Medicina e 12 no D epartamento de H ematologia da Santa Casa 
da M isericórdia de São Paulo, em São Paulo. Cinqüenta e nove casos foram obtidos retrospectivamente através de revisão clínica laboratorial nos quais o diagnóstico prévio de linfoma $T$ ocorreu antes do desenvolvimento do registro. Estes pacientes foram descritos em publicações anteriores. ${ }^{17,18}$ Embora casos isolados da Venezuela, Itália, J apão e G récia tenham sido incluídos na análise (residentes no Brasil há mais de 20 anos), todos os casos foram analisados por local de nascimento para definirmos as diferenças regionais da LLCTA comparadas à prevalência do H TLV-I em cada região. ${ }^{5} \mathrm{Um}$ estudo detransmissão de H TLV-I foi realizado após o diagnóstico da doença e a alta prevalência da infecção (38\%) entre os familiares demonstrou a eficiência da transmissão viral intrafamiliar. ${ }^{19}$

Análise global dos casos demonstrou que as características clínicas de LLcTA no Brasil são semelhantes aos casos descritos no Japão e $C$ aribe, com distribuição igual quanto ao sexo (homem/mulher 1:1), bem como, quanto à freqüência dos subtipos da doença ao diagnóstico. ${ }^{20,21}$ Predominaram as formas agudas da doença ( $60 \%$ ), seguidas de linfoma $(22 \%)$, crônico (10\%) e smoldering $(8 \%)$ na análise dos casos registrados. Os aspectos clínicos laboratoriais mais importantes estão descritos na Tabela 2 e se caracterizaram por linfadenopatia (83\%), hepatomegalia (60\%), lesões de pele (55\%) e hipercalcemia (56\%). Alguns casos apresentaram formas atípicas da doença com lesões tumorais isoladas ou perfil imunofenotípico caracterizado por CD 4-/ CD 8- ou CD 4-/CD 8+. Porém as diferenças marcantes na série de casos brasileiros são a freqüência de LLCTA em crianças e adulto jovens, a idade média dos pacientes de 40 anos (2-94 anos), demonstrando um curto período de latência da infecção viral para transformação em doença maligna. ${ }^{22} 0$ utro achado importante da coorte brasileira foi a presença de 17 pacientes com TSP/H AM ao diagnóstico ou ocorrendo durante o curso clínico da LLCTA. ${ }^{5}$
Tabela 2. Aspectos clínicos e laboratoriais de LLcTA no Brasil.

\begin{tabular}{|c|c|c|c|c|c|}
\hline \multirow{3}{*}{$\begin{array}{l}\text { Características clinicas } \\
\text { elaboratoriais }\end{array}$} & & \multicolumn{4}{|c|}{ Subtipos de LLcTA } \\
\hline & Total & Aguda & Linfoma & Crônica & Smoldering \\
\hline & (\%) & (\%) & (\%) & (\%) & (\%) \\
\hline adenopatia & 83 & 88 & 98 & 60 & 42 \\
\hline es de pele & 55 & 60 & 30 & 73 & 67 \\
\hline enomegalia & 53 & 64 & 54 & 20 & 8 \\
\hline atomegalia & 60 & 77 & 48 & 20 & 17 \\
\hline rcalcemia (1) & 56 & 67 & 50 & $33^{\prime \prime \prime}$ & $12^{11 !}$ \\
\hline ração de SNC (III) & 26 & 27 & 24 & $7^{(111)}$ & $50^{(11)}$ \\
\hline raçâo de $M O$ & 44 & 63 & 18 & 20 & 0 \\
\hline ometria $\left(>10 \times 10^{6} / \mathrm{ml}\right)$ & 73 & 91 & 21 & 100 & 50 \\
\hline citos $\left(>4 \times 10^{6} / \mathrm{ml}\right)$ & 73 & 91 & 12 & 100 & 42 \\
\hline$+(>50 \%)$ & 91 & 94 & 87 & 100 & 67 \\
\hline $5+(>15 \%)$ & 84 & 85 & 77 & 91 & 75 \\
\hline \multicolumn{6}{|l|}{ ão Histológico } \\
\hline pleomórfico & 37 & 38 & 56 & 13 & 8 \\
\hline \multirow{2}{*}{ de pequenas células } & 9 & 8 & 21 & 0 & 0 \\
\hline & 13 & 3 & 0 & 53 & 67 \\
\hline A-Ki+ & 5 & 7 & 6 & 0 & 0 \\
\hline as & 36 & 35 & 22 & 0 & 0 \\
\hline
\end{tabular}

Abreviações: casos que evoluíram com hipercalcemia após diagnóstico; incluindo os 17 pacientes com TSP/H AM concomitante a LLCTA; MF = micose fungóide; $\operatorname{LgCA}=$ linfoma de grandes células anaplásico Ki-1 positive; LN H = linfoma não-H odgkin; LCT = linfoma T; SN C = sistema nervoso central; As percentagens nas colunas dos subtipos de LLCTa referem-se ao total de pacientes em cada grupo, portanto o somatório dos vários grupos não é $100 \%$ e não há correlação com a coluna do total de pacientes.

Devido à diversidade genética da população brasileira, esperávamos encontrar diferenças regionais, como por exemplo, 0 predomínio do subtipo linfoma versus leucemia. Estudo comparativo entre LLCTA no Japão e Jamaica (região caribenha) demonstrou que a forma mais comum da doença na Jamaica é a linfomatosa (65\%), enquanto no Japão predominam as formas leucêmicas (aguda e crônica). ${ }^{23} \mathrm{~N}$ ossa coorte não demonstrou diferenças apesar das influências raciais regionais, como descendentes africanos na Bahia e imigração japonesa em São Paulo. ${ }^{5}$ Ainda no contexto de análise regional, um fato interessante foi a maior incidência de estrongiloidíase nos pacientes da Bahia e Pernambuco versus Rio de Janeiro e São Paulo (p.001). Estrongiloidíase é freqüentemente encontrada em portadores do HTLV-I devido ao estado de imunodeficiência. $\mathrm{Na} \quad \mathrm{M}$ artinica, indivíduos H TLV-I + e com infestação por Strongyloides stercoralis apresentam um padrão de integração monoclonal do HTLV-I proviral nas células linfóides, compatível com a existência de clone de células malignas antes 
mesmo do quadro clinico de LLCTA. ${ }^{24}$

Os diversos aspectos biológicos relacionados à patogênese da infecção por HTLV-I em pacientes brasileiros já foram abordados, tanto no âmbito do hospedeiro quanto do conhecimento da filogenia viral. $4,5,19,22,25$

Apesar do grande potencial numérico de casos de LLCTA para elaboração de condutas terapêuticas diferenciadas dos demais linfomas e leucemias, até o presente, nenhum protocolo inovador foi proposto para ser realizado no Brasil. Por exemplo, as drogas mediadoras do ciclo celular como ácido retinóico, induzem as células de LLCTA à apoptose (ensaios in vitro) e podem reduzir a resistência e estimular o sistema imune no restabelecimento da atividade anti-tumoral do indivíduo contra as células malignas. Alternativamente, a ação de anti-receptores de interleucina ${ }^{2}$ (anti-CD 25) pode ser uma estratégia bem sucedida e merece ser investigada. O s resultados encorajadores com agentes anti-retrovirais e novas drogas biológicas nos estimulam ao debate sobre 0 tema.

\section{REVISÃO DE TRATAMENTO}

Enquanto as diversas modalidades de tratamento conferem grande sucesso nas abordagens de leucemias agudas e linfomas de Hodgkin, o tratamento da LLCTA está aquém do ideal. Os fatores associados à falência do tratamento são resistência à quimioterapia convencional, aumento de proteínas responsáveis pela resistência a múltiplas drogas (MDR), mutação ou inativação da P53, aumento da expressão do bcl-2/ bcl-1, mutação da TAX do HTLV-I, aumento do nível de tioredoxina e estado de imunodeficiência levando a infecções bacterianas, parasitárias e fúngicas no decorrer do tratamento quimioterápico. ${ }^{26}$ Pacientes com as formas crônica e smoldering da LLCTA têm um curso clínico arrastado, com sintomatologia indolente até ocorrer a progressão da doença para forma aguda. Infelizmente não existem evidências de que 0 tratamento quimioterápico precoce vai evitar a transformação aguda. Ao contrário, devido à imunodeficiência, o tratamento poderá aumentar a morbidade com episódios de infecções oportunísticas e resistência aos diversos quimioterápicos na fase aguda da doença.

LLCTA dos tipos agudo e linfoma são reconhecidamente as formas mais agressivas da doença com prognóstico sombrio e sobrevida média de 5-13 meses. 0 s principais fatores prognósticos para a LLCTA são o PS inicial do paciente, idade, o número total de lesões envolvidas, os níveis de cálcio e deD H L séricos. ${ }^{8}$ As principais complicações secundárias ao tratamento caracterizam-se pelo estado de imunodeficiência dos pacientes com: infecção de pele por váriosfungos, pneumonia por P. carinii, herpes zoster, pneumonia intersticial, meningite criptocócica e exacerbação de estrongiloidíase. Baseado em sintomas associados à imunodeficiência os pacientes devem ser estratificados em baixo risco, alto risco e alto risco extremo antes do início do tratamento. ${ }^{8,13}$ A ções corretivas quanto à hipercalcemia se fazem necessárias, com hiper-hidratação, uso de bifosfanatos e calcitonina, bem como tratamento preventivo para fungos, parasitas e infecções virais. Baixas doses de cotrimoxazol, aciclovir oral e antifúngico oral são recomendadas em conjunto com os quimioterápicos.

U ma revisão sobre as diversas formas de tratamentos descritos na literatura estão apresentados na Tabela 3. D evido à alta incidência de LLCTA entre as doenças linfoproliferativas no Japão, os protocolos japoneses apresentam o maior número de casos analisados e portanto resultados mais convincentes. ${ }^{27,28} \mathrm{Na}$ Universidade de Kawasaki, a estratégia utilizada foi 0 uso de CHOP (ciclofosfamida, doxorrubicina, vincristina e prednisona) seguido de etoposídeo, vindesina, ranimustina e mitoxantrone com fator estimulador de crescimento de colônias de granulócitos (G CSF). O s pacientes receberam 2-4 cursos de quimioterapia com intervalos de 3 semanas. Aqueles que apresentaram remissão completa 
(RC) ou parcial (RP) recebiam etoposídeo $\left(50 \mathrm{mg} / \mathrm{m}^{2}\right)$ como tratamento de manutenção. 0 resultado deste protocolo foi superior ao uso anterior de metotrexate, doxorrubicina, ciclofosfamida, vincristina, prednisona e bleomicina (CHOP, MACOP-B) ou vincristina, ciclofosfamida, prednisona e doxorrubicina (VEPA), com 35,8\% de RC e 38,3\% RP na série de 83 pacientes elegíveis para o estudo. As principais toxicidades deste esquema foram pancitopenia, neurite periférica e alopecia. ${ }^{27}$ Etoposídeo em baixas doses ( $50 \mathrm{mg} / \mathrm{dia}$ ) foi relatado em uma paciente cujo estado clínico não permitia o uso de quimioterapia convencional. H ouve remissão completa do quadro sem reações adversas graves, com sobrevida de aproximadamente dois anos. ${ }^{29} \mathrm{~N}$ o entanto, apesar da introdução de etoposídeo, a taxa de sobrevida mediana não ultrapassou nove meses para grande maioria dos casos e de 3 anos para 13\% dos casos.

Tabela 3. Resultados de estudos de tratamento da ATL.

\begin{tabular}{|c|c|c|c|c|c|}
\hline$\#$ & REFERÊNCIA & LOCAL & $v$ & REGIME & SOBREVIDA \\
\hline 1 & Taguchi $\mathrm{H}^{27}$ & Japão & 83 & $\begin{array}{l}\text { VCR, ADM, CPA-DI } \\
\text { ETP- DI-8 } \\
\text { PDNL- DI-3D8-10 } \\
\text { MCNU, VDS, Mitox-D8 } \\
\text { G-CSF- D 9-21 }\end{array}$ & $\begin{array}{l}\mathrm{SM}=8,5 \mathrm{~m} \\
\mathrm{SLD}=7,6 \mathrm{~m}\end{array}$ \\
\hline 2 & $\begin{array}{l}\text { Bazarbachi \& } \\
\text { Hermine }^{33}\end{array}$ & França & 10 & $\begin{array}{l}\text { AZT }=1 \mathrm{~g} / \text { dia } \\
\text { alFN }=9 \text { milhões } \mathrm{U} / \text { dia }\end{array}$ & $\begin{array}{l}\text { SLE }=12 \mathrm{~m} \\
\mathrm{SG}=15 \mathrm{~m}\end{array}$ \\
\hline 3 & Gill $p^{34}$ & EUA & 19 & $\begin{array}{l}\text { AZT }=1,2 \mathrm{~g} / \text { dia } \\
\text { alFN }=5-15 \text { milhöes U/dia }\end{array}$ & $\begin{array}{l}\mathrm{SG}=3 \text { meses } \\
\mathrm{SLL}=13 \text { meses }\end{array}$ \\
\hline 4 & Tajima K ${ }^{31}$ & Japão & 1 & TMO alogênico & $S G=24$ meses \\
\hline 5 & Maeda $Y^{40}$ & Japão & 1 & ATRA $=60 \mathrm{mg} /$ dia VO & PCR(-)após \\
\hline 6 & White $\mathrm{JD}^{37}$ & Jamaica & 18 & $\begin{array}{l}\alpha \text { IFN }=2,5-10 \mathrm{~m} / \mathrm{USC} \\
\text { AZT } 1 \mathrm{~g} / \mathrm{dia} \mathrm{VO}(12 \mathrm{~m})\end{array}$ & $S G=6 \mathrm{~m}$ \\
\hline 7 & Yamada Y 28 & Japão & 96 & $\begin{array}{l}\text { VCAP; AMP } \\
\text { VECP; G-CSF -7 ciclos }\end{array}$ & $\begin{array}{l}\mathrm{SM}=13 \text { meses } \\
\mathrm{SG}=31,3 \% \\
24 \mathrm{~m}\end{array}$ \\
\hline 8 & Xiniia $^{29}$ & Japão & 1 & $\mathrm{ETP}=50 \mathrm{mg} / \mathrm{dia}$ & $S G=13 \mathrm{~m}$ \\
\hline 9 & Matutes $\mathrm{E}^{36}$ & Inglaterra & 15 & $\begin{array}{l}\text { AZT lg/dia } \\
\text { alFN 3-5 milhöes U/dia }\end{array}$ & $S M=18 \mathrm{~m}$ \\
\hline
\end{tabular}

Abreviações: $n=$ numero de casos; SLE $=$ sobrevida livre de eventos; SG = sobrevida global; SM = sobrevida mediana; $\mathrm{SLD}=$ sobrevida livre de doença; $\mathrm{TMO}=$ transplante de medula óssea; ICT = irradiação corporal total; VCR = vincristina; $C P A=$ ciclofosfamida; $A D M=$ doxorrubicina; $\mathrm{PDN}=$ prednisona; $\mathrm{MCNU}=$ ranimustina; $\mathrm{VDS}=$ vindesina; $E T P=$ etoposídeo; $M$ itox = mitoxantrone; $A Z T$ = zidovudina; al N F = interferon alfa 2b; ATRA = ácido transretinóico; VECP = vindesina, etoposideo, carboplatina e prednisona; $A M P=$ doxorrubicina, ranimustina $e$ prednisona; VCAP = vincristina, ciclofosfamida, doxorrubicina e prednisona; $\mathrm{G}-\mathrm{CSF}=$ fator de crescimento granulocítico.
Embora novas estratégias terapêuticas utilizando drogas moduladoras biológicas e anti-retrovirais, o G rupo Japonês para Estudo de Linfomas insiste na poliquimioterapia intensa com uso de fatores estimuladores de colônias de crescimento de granulócitos e macrófagos (GM-CSF) para evitar perdas devido aos efeitos colaterais como infecções e falência medular no tratamento da LLCTA. ${ }^{28}$ O s resultados do protocolo LSG 15 utilizando o esquema vincristina, ciclofosfamida, doxorrubicina e prednisona (VCAP) seguido de doxorrubicina, ranimustina e prednisona (AM P) e vindesina, etoposídeo, carboplatina e prednisona (VECP) são semelhantes aos do protocolo de Kawasaki quanto às taxas de RC e RP, com discreto aumento na sobrevida em $31 \%$ dos casos. Casos isolados de tratamento com transplante de medula óssea (T M O) alogênico, foram relatados, porém a casuística é pequena e o tempo de acompanhamento ainda é insuficiente para conclusão definitiva quanto a eficácia deste procedimento. Borg et $a^{30}$ e Tajima et al ${ }^{31}$ relataram casos isolados do LLcTA submetidos a T M 0 com remissão completa mantida de 23 e 24 meses respectivamente. Talvez a maior experiência no tratamento do LLCTA com TMO seja de $U$ tsunomiya et al,32 que descreveram os resultados não muito promissores de 10 pacientes submetidos a TM O . A mortalidade de $40 \%$ dos casos e $20 \%$ de recaída com sobrevida livre de linfoma (SLL) de 17 meses, demonstra a necessidade de aprimorar essa opção terapêutica.

0 tratamento mais atrativo no momento, devido a sua baixa toxicidade e aos bons efeitos em causar remissão e prolongar a sobrevida para LLCTA é o uso de zidovudina (AZT) combinado ao interferon ( $\alpha$ I N F-2b). ${ }^{33,34}$ Embora o verdadeiro mecanismo de ação seja desconhecido, especula-se que haja um efeito imunológico do $\alpha$ INF associado ao efeito citostático do AZT nas células linfóides. No entanto, Bazarbachi e $\mathrm{Hermine}^{35}$ não demonstraram efeito citotóxico direto desta combinação nos linfócitos $T$ infectados in vitro, ao contrário do que é descrito na 
infecção por vírus da imunodeficiência humana. Apesar destas diferenças entre ensaios in vitro e ex vivo, os resultados encorajadores com agentes anti-retrovirais em LLcTA são motivo de pesquisa para o ajuste de esquema com novas drogas biológicas. A dose preconizada para o uso do AZT é de $1 \mathrm{~g} /$ dia, via oral, em doses fracionadas. 0 interferon ( $\alpha \mid N F-2 b)$ é utilizado em doses variadas de 3 a 6 milhões U/dia, via subcutânea de acordo com a tolerância de cada individuo. O s efeitos colaterais são principalmente sintomas gripais, depressão, alopecia e neutropenia. $\mathrm{N}$ a série de casos descritos por $M$ atutes et al, ${ }^{36}$ a estratégia utilizada foi diminuir o volume da doença com poliquimioterapia ( 3 ciclos) e em seguida utilizar AZT e $\alpha$ INF como continuação do tratamento. A sobrevida mediana foi de 18 meses, com ressalva que os pacientes responsivos à quimioterapia inicial tiveram sobrevida significativamente superior aos não respondedores ( 6 meses). Este esquema mostrou-se superior aos estudos anteriores que não associaram quimioterapia prévia ao AZT e $\alpha$ IN F eàquelescom poliquimioterapia sem dose de manutenção. ${ }^{37}$

A necessidade de quimioterapia convencional antes do uso do AZT/IFN para reduzir a carga tumoral ainda precisa ser estabelecida, pois nenhum estudo apresenta uma casuística homogênea. Em todos os estudos mencionados, houve a inclusão de casos de LLCTA crônica e smoldering que naturalmente tem curso clínico arrastado independente de qualquer tratamento, juntamente com subtipos agudos que são as formas agressivas da doença.

$Q$ uando analisamos os resultados de todas as modalidades terapêuticas até hoje empregadas no tratamento de LICTA, nenhum protocolo é satisfatório (Figura 2). N ão há tratamento curativo ea sobrevida éprolongada em apenas al guns meses. Conforme abordado anteriormente, os fatores associados à falência dos tratamentos são resistência à quimioterapia convencional e o aumento de proteínas responsáveis pela MDR, demonstrado pelas expressões da glicoproteína P (MDR-1), pela expressão de RNA-m da proteína de resistência a múltiplas drogas (MRP) e pela proteína de resistência pulmonar (LRP). ${ }^{26,38}$ Para tentar reverter este quadro, novas drogas têm sido utilizadas como possibilidade terapêutica para a LLcTA. 0 ácido transretinóico (ATRA) que inibe o crescimento das células da LLCTA in vitro e induz apoptose suprimindo a atividade de transcrição da proteína N F-kB no ciclo celular, ${ }^{39}$ foi utilizado por M iyatake para tratamento de um paciente com LLCTA refratária, na dose de $60 \mathrm{mg} /$ dia VO com remissão completa do quadro..$^{38-43} \mathrm{O}$ utro modificador biológico é o trióxido de arsênico, que inibe o crescimento e induz apoptose das células infectadas pelo H TLV-I através da ativação da via das carspases e pela inativação da via N F-kB. Esta droga deve ser utilizada em associação com $\alpha$ IN F ou ATRA, pois estudos sugerem que seu uso isoladamente poderia aumentar a produção viral e 0 número de células infectadas. ${ }^{43,44}$

Figura 2. Respostas parcial (RP) e completa (RC) dos pacientes aos principais esquemas terapêuticos presentes na Tabela 3.

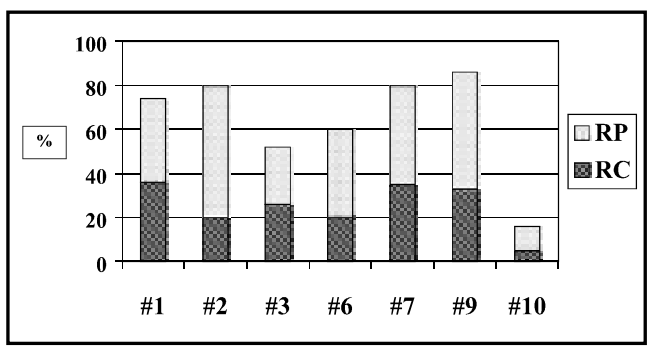

A imunoterapia com anticorpo monoclonal anti-CD 25 (H AT, Zenapax) também desponta como uma opção promissora no tratamento de LLCTA. N a série de 19 pacientes tratados com HAT, 4 permaneceram em RP e alcançaram 2 RC, com sobrevida de 12 meses. ${ }^{44}$ Ressaltamos que estes resultados são provenientes de ensaio clínico fase II, onde os pacientes incluídos foram refratários aos esquemas quimioterápicos convencionais e com PS alto. ${ }^{44,45}$ HAT foi testado em altas 
doses (>1mg/kg) sendo demonstrado, saturação $\geq 95 \%$ do CD 25 com dose de $6 \mathrm{mg} / \mathrm{kg}{ }^{45}$

Até o momento não foi estabelecido qual o melhor tratamento para a LLCTA com drogas que interferem na dinâmica da patogênese da doença. $0 \mathrm{~s}$ estudos realizados foram baseados em casuísticas pequenas, que impedem a randomização por subtipos de LLCTA e não apresentam grupo controle para comparação entre fase crônica e aguda. Isto se deve à baixa prevalência da infecção por H TLV-I e LLCTA nos países que lideram as pesquisas clínicas. No Brasil novos estudos se fazem necessários para melhorar a sobrevida destes pacientes. A incidência de casos LLCTA demonstrada através de estudo epidemiológico recente, irá nos permitir elaborar propostas terapêuticas semelhantes àquelas realizadas no J apão com mais de 80 pacientes. Através de um estudo cooperativo nacional seremos capazes de melhorar a sobrevida dos pacientes e dar continuidade às pesquisas sobre patogênese de LLCTA.

\section{REFERÊN CIAS BIBLIO G RÁFICAS}

1. Blattner WA, Pombo de O liveira M S. HTLV-I and H TLV-II. In: M origan TC, Bartlett JC, Bolognesi $D$, editors. Textbook of AID S M edicine. 2nd ed. Baltimore: W illiams\& W ilkins; 1999. p. 1003-29.

2. $M$ anns $A, H$ isada $M, D$ a G renadeL. $H$ uman T- lymphotropic virustypel infection. Lancet 1999;353:1951-8.

3. Galvão-Castro $B, L$ oures $L$, RodriquesLG, Sereno A, Ferreirajúnior OC, Franco LG , etal. D istribution of human T-lymphotropic virustypel among blood donors: anationwidebrazilian study. Trans fusion 1997;37(2):242-3.

4. Farias de $C$ arvalho SM F, Pombo de O liveira MS, Thuler LC, Rios M, C oelho RC, Rubim LC. H T LV-I and H TLV-II infectionsin hematological disorder patients, cancer patients, and healthy individualsfrom Rio de aneiro, Brazil. J Acquir ImmuneD efic Syndr H um Retrovirol 1997;15(3):238-2.

5. Pombo deO liveiraM $S$, Loureiro $P$, Bittencourt A, C hiattone C, Borducchi D, D e Carvalho SM, et al. G eographic diversity of adultT-cell leukemia/lymphoma in Brazil. Int J Cancer 1999;83(3):291-8.
6. Shimoyama M . D iagnostic criteria and classification of clinical subtypes of adult T-cell leukemia-lymphoma. A report from the Lymphoma Study G roup. Br J H aematol 1991;79:428-7.

7. Tachibana N, O kayama A, IshiharaS, Schioiri S, M urai K, TsudaK, et al. High H TLV-I proviral D N A level associated with abnormal lymphocytes in peripheral blood from asymptomatic carriers. Int J C ancer 1992;51:593-5.

8. Levine PH, Cleghorn $F, M$ anns $A$, Jaffe $E S$, N avarro-Roman L, Blattner WA, et al. Adult Tcell leukemia/lymphoma: aworking point-score classification for epidemiological studies. Int J Cancer 1994;59:491-3.

9. Lymphoma Study G roup. M ajor prognostic factors of patients with adultT cell leukemial Iymphoma: a cooperative study. Leuk Res 1991;15:81-90.

10. Yamada Y, O hmoto $Y, H$ ataT, Yamamura M , M urata K, Tsukasaki K, et al. Features of the cytokines secreted by adult $T$ cell leukemia (ATL) cells. Leuk Lymphoma 1996;21(5/ 6):443-7.

11. Kamihira S, Sohda H , Atogami S, Toriya K, Yamada Y, Tsukazaki K, et al. Phenotypical diversity and prognosis of adult T-cell leukemia. Leuk Res 1992;16(5):435-9.

12. Shirono K, H attori T, Takatsuki K. A new classification of clinical stages of adult T-cell leukemia based on prognosis of the disease. Leukemia 1994;8(11):1834-7.

13. O hshima K, Suzumiya J, Sato K, Kanda M, Sugihara M , H araokaS, et al. N odal T-cell lymphoma in an H TLV-I-endemic area: proviral H TLV-I D N A, histological classification and clinical evaluation. $\mathrm{Br} J \mathrm{H}$ aematol 1998;101(4):703-11.

14. Yoshida M , Seiki M , Yamaguchi K, Takatsuki K. M onoclonal integration of human T-cell leukemia provirusin all primary tumors of adult T-cell leukemia suggests causative role of human T-cell leukemia virusin the disease. Proc N atl Acad Sci U SA 1984;81(8):2534-7.

15. Shimamoto $Y$, Kobayashi M, M iyamoto $Y$. Clinical implication of theintegration patterns of human T-cell lymphotropic virus type I proviral DN A in adult T-cell leukemia/lymphoma. Leuk Lymphoma 1996;20:207-15.

16. TamiyaS, M atsuoka M , Etoh $\mathrm{K}$, WatanabeT, KamihiraS, Yamaguchi K, et al. Two types of defective human T-lymphotropic virus typel provirus in adult T-cell leukemia. Blood 1996;88:3065-73.

17. Pombo de Oliveira M S, M atutes E, Schulz T, 
Carvalho SM F, N oronhaH , ReavesJ D, et al. Tcell malignancies in Brazil. C linicopathological and molecular studies of H T LV-I positiveand negativecases. Int J Cancer 1995;60(6):823-7.

18. Bittencourt $A$, BarbosaH S, Brites $C$, Ferraz $N$, Pereira Filho CS, $\mathrm{H}$ arrington $\mathrm{W}$. Clinocopathological aspects of H T LV-I positiveand negativecutaneousT-cell lymphoma. Eur J Dermatol 1997;7:283-9.

19. Pombo de O liveira M S, Carvalho SM C, Borducchi D, D obbin J, Salvador J, CorreaRB, et al. AdultT-cell leukemia/lymphoma and cluster of H T LV-I associated diseasesin brazilian settings. Leuk Lymphoma 2001;42(1/2):135-44.

20. Takatsuki K, M atsuokaM , Yamaguchi K. Adult T-cell leukemia/lymphoma in Japan. J Acquir ImmuneD efic Syndr H um Retrovirol 1996;13 Suppl 1:s15-9.

21. Gerard Y, Lepere JF, Fradinaud R, Joly F, Lepelletier L, Joubert M , et al. Clustering and clinical diversity of adultT-cell leukemia/lymphoma associated with HTLV-I in a remote black population of French $\mathrm{G}$ uiana. Int J $\mathrm{C}$ ancer 1995;60:773-6.

22. Pombo deO liveira M S, D obbin J, Loureiro $P$, Borducchi D , M aiaRC, Fernandes M A, et al. $G$ enetic mutation and early onset of T-cell leukemiain pediatric patientsinfected at birth with H TLV-I. Leuk Res 2002;26:155-61.

23. Cleghorn $F, M$ anns $A$, Falk $R, H$ artge $P$, $H$ anchard B, Jack N , et al. Effect of H uman TLymphotropic Virus type-infection on non$\mathrm{H}$ odgkin'slymphomaincidence. J $\mathrm{N}$ atl $\mathrm{C}$ ancer Inst 1995;87(13):1009-14.

24. Watel $E$, Vartanian JP, Pannetier $C$, Wain$\mathrm{H}$ oleson S. Clonal expression of H T LV-I infected cells in asymptomatic and symptomatic carrier without malignancy. J Virol 1999;69:2863-8.

25. Sambor AM , Pombo deO liveira M S, Farhadi A, CarrJK, Carvalho SM , Blattner WA, et al. Human t-lymphotropic virus typel tax polymorphismsin atransmission cohort: no association between sequence variation and disease manifestations. J H um V irol 1999;2(5):308-14.

26. Bazarbachi $A$, H ermineO . Treatment of adultTcell leukaemia/lymphoma: current strategy and futureperspective. VirusRes2002;78:79-92.

27. Taguchi $H$, Kinoshita K, Takatsuki K, Tomonaga M , Araki K, I kedaS, et al. An intensivechemoterapy of adultT-cell leukemia/lymphoma: CHOP followed by etoposide, vindesine, ranimustine, and mitoxantronewith granulocytecolony-stimulating factor support.
J Acquir ImmuneD eficSyndr H um Retrovirol 1996:12:182-6.

28. YamadaY, Tomanaga M , FukudaH , H anadaS, U tsunomiya A, Tara M , et al. A new G-CSFsupported combination chemotherapy, LSG 15, for adultT-cell leukemia/lymphoma: Japan clinical oncology group study $9303 . \mathrm{Br}$ J H aematol 2001;113:375-82.

29. Xinjia F, M atano S, Amitani S, Terahata S, Furusyo $\mathrm{H}$, Yamamoto $M$, et al. Low-dose etoposidein a patient with adultT-cell leukemia/lymphoma who had severecomplications. Gan To Kagaku Ryoho 2001;28:1269-72.

30. Borg A, Yin L, Johnson PRE, Tosswili J, Saunders M , M orrisD. Succesful treatment of H T LV-I associated acute adultT-cell leukemia lymphoma by allogeneic bone marrow transplantation. BrJ H aematol 1996;94:713-5.

31. TajimaK, AmakawaR, U ehiraK, M atsumoto $N$, Shimizu T, M iyazaki Y, et al. Adult T-cell leukemia successfully treated with allogeneic bonemarrow transplantation. Int J H aematol 2000;71(3):290-3.

32. Utsunomiya A, M iyazaki $Y$, Takatsuka $Y$, $\mathrm{H}$ anadaS, U ozumi K, Yashiki S, et al. Improved outcome of adultT cell leukemia/lymphoma with allogeneic hematopoietic stem cell transplantation. Bone M arow Transplant 2001;27(1):15-20.

33. Bazarbachi A, H ermine O . Treatment with a combination of zidovudineand alfa-interferon in naive and pretreated adultT-cell leukemia/ lymphoma patients. J Acquir Immune D efic Syndr H um Retrovirol 1996;13(Suppl 1):S186-90.

34. Gill PS, H arrington W, Kaplan M H , Ribeiro R, Bennett JM, et al. Treatment of adult T-cell leukemia/lymphoma with a combination of interferon alpha and zidovudine. $N$ ew Engl J M ed 1995; 332:1744-8.

35. Bazarbachi A, N asr R, El-Sabban M E, M aheA, $M$ ahieux R, G essain A, et al. Evidenceagainst a direct cytotoxic effect of alpha interferon and zidovudinein H TLV-I associated adultT cell leukemia/lymphoma. Leukemia 2000;14:716-21.

36. M atutes E, Taylor GP, C avenagh J, Pagliuca A, Bareford D , D omingo A, et al. Interferon al pha and zidovudine therapy in adult $\mathrm{T}$-cell leukemia/lymphoma: response and outcomein 15 patients. Br J H aematol 2001;113:779-84.

37. WhiteJD, W harfeG, Stewart D M , M aher VE, Eicher $D, H$ erring $B$, et al. The combination of zidovudineand interferon al pha $2 B$ in thetreatment of adultT-cell leukemia/lymphoma. Leuk 
Lymphoma 2001;40(3/4):287-94.

38. Wang BJ, Kobayashi M, Sakurada K, Inamura $M$, M uriochi T, H osokawa M . Possibleroles of an adultT-cell leukemia (ATL)-derived factor/ thioredoxin in the drug resistance of AT $L$ to adriamycin. Blood 1997;7:2480-7.

39. N awata H, M aedaY, Sumimoto Y, M iyatakeJ, Kanamaru A. A mechanism of apoptosis induced by of all-trans retinoic acid on adult Tcell leukemia cells: a possible involvement of the Tax/N F-kB signaling pathway. Leuk Res 2001;25:323-31.

40. M aeda $Y, N$ aiki $Y$, Sono H, M iyatake J, Sumimoto Y, Sakaguchi M , et al. C linical application of all-trans retinoic acid (tretinoin) for adult $\mathrm{T}$-cell leukemia. $\mathrm{Br} \mathrm{J} \mathrm{H}$ aematol 2000;109:677.

41. M iyatakeJ I, M aedaY. Inhibition of proliferation and $C D 25$ down-regulation by retinoic acid in human adult T cell leukemia cells. Leukemia 1997;11:401-7.

42. El-Sabban M E, N asr R, D baibo G , H ermine
O, Abboushi N, Q uignon F, et al. Arsenic-interferon-alpha-triggers apoptosis in H T LV-I transformed cellsisassociated with Tax downregulation and reversal of N F-kB activation. Blood 2000;96:2849-55.

43. M ahieux R, Pise-M asison C, Brady J, Gessain A, Yamaoka S, Franchini G. Arsenic trioxide induces apoptosis in human T-cell leukemia virustypel and typell infected cells by a caspase 3-dependent mechanism involving Bcl-2 cleavage. Blood 2001;98(13):3762-9.

44. WaldmannTA.T-cell receptorsfor cytokines: targetsfor immunotherapy of leukemia/lymphoma. Ann 0 ncol 2000;11(Suppl 1):S101-6.

45. M orris]C, JanickJE, Turner M , LeC, Top L, Jaffe ES. A phasel/II study of theefficacy and toxicity of humanized anti-tac (H AT, Zenapaz) for thetreatment of tac, expressing human lymphotropic virus typel (H TLV-I)-associated adultT-cell leukemial lymphoma(ATL): interim results. AID SResH um Retrovirol 2001;17(Suppl1):S29." 